\title{
Determinants of high sensitivity troponin $T$ concentration in chronic stable patients with heart failure: Ischemic heart failure versus non-ischemic dilated cardiomyopathy
}

\author{
Ruken Bengi Bakal ${ }^{1}$, Suzan Hatipoglu ${ }^{1}$, Gokhan Kahveci ${ }^{1}$, \\ Mehmet Onur Omaygenc ${ }^{1}$, Tuba Unkun ${ }^{1}$, Taylan Akgun ${ }^{1}$, \\ Muslum Sahin ${ }^{1}$, Ali Elveran ${ }^{2}$, Olcay Ozveren ${ }^{3}$, Nihal Ozdemir ${ }^{1}$ \\ ${ }^{1}$ Kartal Kosuyolu Heart and Research Hospital, Istanbul, Turkey \\ ${ }^{2}$ Yeditepe University Hospital, Istanbul, Turkey \\ ${ }^{3}$ Gebze Darıca Farabi State Hospital, Kocaeli, Turkey
}

\begin{abstract}
Background: Cardiac troponin $T$ is a marker of myocardial injury, especially when measured by means of the high-sensitivity assay (hs-cTnT). The echocardiographic and clinical predictors of hs-cTnT may be different in ischemic heart failure (IHF) and non-ischemic dilated cardiomyopathy (DCM).
\end{abstract}

Methods: Sixty consecutive patients (19 female, 41 male; mean age $56.3 \pm 13.9$ years) with stable congestive heart failure (33 patient with IHF and 27 patients with DCM), with New York Heart Association functional class I-II symptoms, and left ventricular ejection fraction $<40 \%$ were included.

Results: In patients with IHF peak early mitral inflow velocity (E), E/peak early diastolic mitral annular tissue Doppler velocity (Em) lateral, peak systolic mitral annular tissue Doppler velocity (Sm) lateral and $\operatorname{logBNP}$ were univariate predictors of hs-cTnT above median. But only E/Em lateral was an independent predictor of hs-cTnT above median ( $p=0.04, H R: 1.2$, CI: 1-1.4). In patients with DCM; left atrial volume index, male sex, Sm lateral and global longitudinal strain (LV-GLS) were included in multivariate model and LV-GLS was detected to be an independent predictor for hs-cTnT above median ( $p<0.05, H R: 0.7, C I: 0.4-1.0)$.

Conclusions: While LV-GLS is an independent predictor of hs-cTnT concentrations in patients with DCM, E/Em lateral predicted hs-TnT concentrations in patients with IHF. (Cardiol J 2014; 21, 1: 67-75)

Key words: high sensitivity troponin T, myocardial mechanics, heart failure, echocardiography

Address for correspondence: Ruken Bengi Bakal, MD, Kartal Kosuyolu Heart and Research Hospital, Department of Cardiology, Denizer Caddesi, 34846, Kartal/Istanbul, Turkey, tel: +90 5334147788 , fax: +90 2164596321 , e-mail: rukenbengi@gmail.com

Received: 20.03.2013 Accepted: 15.05.2013 


\section{Introduction}

Conventional cardiac troponin $\mathrm{T}$ (cTnT) assays have often been used as a negative or positive categorical variable especially to detect acute coronary syndromes [1]. But recently, stepwise rises in high sensitivity cardiac troponin T (hs-cTnT) in patients with chronic heart failure (HF) have been associated with adverse cardiac events [2]. Several observations have been made in the general population. Hs-cTnT at baseline and during follow-up is a powerful predictor of cardiac events in patients with $\mathrm{HF}$ and in the general population $[3,4]$.

Natriuretic peptide concentrations have been shown to parallel with the clinical severity of congestive $\mathrm{HF}$ and cardiac dysfunction [5]. B-type natriuretic peptide (BNP) may be viewed as a marker of ventricular load and hs-c TnT as marker of myocardial injury [6].

The risk stratification of patients with left ventricular (LV) dysfunction can also be performed using echocardiographic parameters such as the ejection fraction (EF) and LV global longitudinal peak systolic strain (LV-GLS). LV-GLS is highly feasible and reliable in patients with LV dysfunction and provides incremental prognostic value to $\mathrm{EF}[7,8]$.

The etiology of HF has been studied extensively with regard to prognosis and ischemic HF (IHF) was associated with adverse survival in many studies $[9,10]$.

Even if the clinical presentation is similar, the pathophysiology and prognosis differ in IHF and non-ischemic dilated cardiomyopathy (DCM), thus echocardiographic and clinical predictors of hs-cTnT concentrations may be different in these two distinct entities. Therefore, the purpose of the study was to evaluate the correlation between hs-cTnT and advanced echocardiographic parameters or BNP.

\section{Methods}

\section{Study population}

Sixty consecutive patients with stable congestive HF (33 patient with IHF and 27 patients with DCM) who were admitted to HF outpatient clinic were enrolled into the study. The inclusion criteria were: New York Heart Association (NYHA) functional class I-II HF symptoms, LVEF $<40 \%$, confirmed ischemic or non-ischemic HF on cardiac catheterisation, normal serum sodium concentrations indicating euvolemic state and stable clinical condition with no hospitalization for HF or acute ischemic event in the past 6 months. Patients with NHYA functional class III-IV symptoms, history of involuntary change of weight $>2 \mathrm{~kg}$ within 4 weeks prior to the outpatient visit, significant renal dysfunction as identified by a creatinine concentration $>2 \mathrm{mg} / \mathrm{dL}$, angina or angina equivalent symptoms in case of ischemic origin were excluded. Blood samples for hs-cTnT measurements were drawn just before the echocardiographic examination. Patients were treated according to the current HF guidelines [11]. The study protocol was approved by the institutional ethical committee and written informed consent was given by all subjects.

\section{Risk factors}

Arterial hypertension was defined as a reported blood pressure of $>140 / 90 \mathrm{~mm} \mathrm{Hg}$ or in patients receiving anti-hypertensive therapy. Diabetes mellitus was defined according to the World Health Organization definition as a fasting blood glucose concentration of $>126$ or $>200 \mathrm{mg} / \mathrm{dL}$ $2 \mathrm{~h}$ after an oral glucose tolerance test or in patients receiving permanent medical anti-diabetic therapy. Hyperlipidemia was defined as blood total cholesterol concentrations of $>180 \mathrm{mg} / \mathrm{dL}$ or low density lipoprotein of $>130 \mathrm{mg} / \mathrm{dL}$ or when patients were receiving permanent treatment with lipid-lowering agents. Impaired renal function was defined as glomerular filtration rate (GFR) of $<60 \mathrm{~mL} / \mathrm{min}$.

\section{Blood samples}

EDTA-treated plasma samples were obtained from trial participants simultaneously with echocardiographic examination and centrifuged at room temperature. The plasma component was aspirated and frozen at $-20^{\circ} \mathrm{C}$. Within 3 months after collection plasma samples were taken to the laboratory on dry ice for evaluation. Plasma hs-cTnT concentrations were measured with hs-cTnT reagents by electrochemiluminescence immunoassay (ECLIA) with Elecsys Troponin T-High Sensitive Immunoassay (Roche Diagnostics Ltd.), with an analytical measurement range of $0.003-10 \mathrm{ng} / \mathrm{mL}$. The value at the $99^{\text {th }}$ percentile cut-off from a healthy reference population was $0.014 \mathrm{ng} / \mathrm{mL}$. Interassay coefficient of variation according to the manufacturer was $3.1 \%$ at $0.024 \mathrm{ng} / \mathrm{mL}$ and $1.3 \%$ at $0.300 \mathrm{ng} / \mathrm{mL}$ [12].

The quantitative determination of BNP in plasma was done by immune fluorescence method using the ADVIA Centaur System (Bayer Diagnostics, Tarrytown, NY, USA). GFR was estimated by age, weight, creatinine, and sex using the Cockcroft-Gault formula. Hemoglobin, sodium, 
potassium and blood urea nitrogen concentrations belonging to the same outpatient clinic visit were also obtained from our local laboratory.

\section{Transthoracic echocardiographic examination}

All echocardiographic examinations and tissue Doppler imaging (TDI) were performed using 2$-4 \mathrm{MHz}$ phased array transducer (Vivid 7 pro; GE, Horten, Norway) by a cardiologist and recorded for offline analysis (Echopac PC, GE). Individuals were instructed to hold their breath, and images were coupled with electrocardiographic recordings. Measurements were done offline later by a single investigator with more than four years' experience, who was blinded to the clinical and hs-cTnT data. M-mode measurements were performed according to the criteria of the American Society of Echocardiography. Three consecutive cycles were averaged for every parameter and 5 cycles for atrial fibrillation. Left atrial (LA) dimension and LV end-systolic and end-diastolic diameters were measured. LVEF was estimated by Simpson's rule. Interventricular septum thickness (IVST) and posterior wall thickness (PWT) were measured, and LV mass (LVM) was estimated. For determination of LVM, the Devereux formula was used: LVM $[\mathrm{g}]=$ $=0.8 \times\left(1.04 \times\left[\left(\right.\right.\right.$ LVID + PWT + IVST $^{3}-$ - LVID $\left.^{3}\right]$ ) + 0.6 ; (LVID indicates LV internal dimension). LVM index (LVMI) was calculated by dividing LVM by body surface area [13].

Left atrial volume (LAV) was calculated at end systole of the left ventricle by measuring the LA area in the apical 4- (A1) and 2-chamber (A2) views and measuring the LA distance from apical 4 chamber view beginning from the center of mitral annulus. A1 × A2 × 0.85/LA distance formula was used to calculate LAV. LA volume index (LAVI) was calculated by dividing LAV by body surface area and expressed in $\mathrm{mL}$ per $\mathrm{m}^{2}$ [14].

Transmitral pulsed-wave Doppler velocities were recorded from the apical 4 -chamber view with the Doppler sample placed between the tips of the mitral leaflets. Early (E) and late (A) wave velocities, $\mathrm{E} / \mathrm{A}$ ratio, $\mathrm{E}$ deceleration time were measured from the mitral inflow profile. The same echocardiography machine was used to acquire TDI data at high frame rates. The Nyquist limit was set at $15-20 \mathrm{~cm} / \mathrm{s}$, and minimal optimal gain was used. The myocardial systolic (Sm), early diastolic (Em), and late diastolic (Am) velocities were obtained at the septal and lateral mitral annulus by placing a sample volume. The E/Em ratio was subsequently calculated for septal and lateral measure- ments. Mitral regurgitation was characterized as follows: mild (regurgitation orifice area $\leq 0.2 \mathrm{~cm}^{2}$ ), moderate $\left(0.2-0.39 \mathrm{~cm}^{2}\right)$ and severe $\left(\geq 0.4 \mathrm{~cm}^{2}\right)$. Intraobserver variability in measurement ranged from $4 \%$ for tissue Doppler indexes to $8 \%$ for transmitral indexes.

\section{LV global longitudinal strain measurements}

The LV-GLS measurements were performed offline using dedicated software (Echopac PC) and 2-dimensional speckle tracking imaging method. Special care was taken to ensure frame rates of between 50 and 90 frames per second in all patients. One cardiac cycle was acquired from apical 4-chamber, 2-chamber and long axis views for LV-GLS; the endocardial border was traced manually in the end-diastolic frame. In segments with poor tracking (assessed subjectively) endocardial borders were readjusted until better tracking was achieved. If this was unattainable, that segment was excluded. Graphical displays of deformation parameters for each segment were then generated automatically and were used for measurement of LV-GLS values (Fig. 1). Each apical view was divided into 6 segments by the software. Peak systolic longitudinal strain was calculated by averaging the peak systolic values of 18 segments, derived from 3 apical views. Peak global strain was defined as the peak negative systolic value on the strain curve during the entire cardiac cycle. We obtained LV-GLS only in the case of adequate tracking quality $>5$ of the 6 segments per view. Any view in which 2 or more segments could not be tracked was not included in the analysis, and the remaining apical views were averaged to calculate LV-GLS. Reproducibility in the LV-GLS measurement was $0.59 \pm 0.70 \%$ and $93 \%$ (1021/1098) of the myocardial segments were adequately tracked.

\section{Statistical analysis}

Continuous variables are summarized as mean \pm SD if normally distributed, and as median if distributed not normally. Also, if plasma hs-cTnT and BNP concentrations were non- normally distributed, we converted these data to log format for various comparisons. Normally distributed variables were compared using t-test and non-normally distributed variables were analyzed with Mann-Whitney U test.

Non-parametric correlations between hs-cTnT concentrations and clinical variables were determined by Spearman's rho test. The patients were grouped according to median hs-cTnT value and binary logistic regression analysis was 


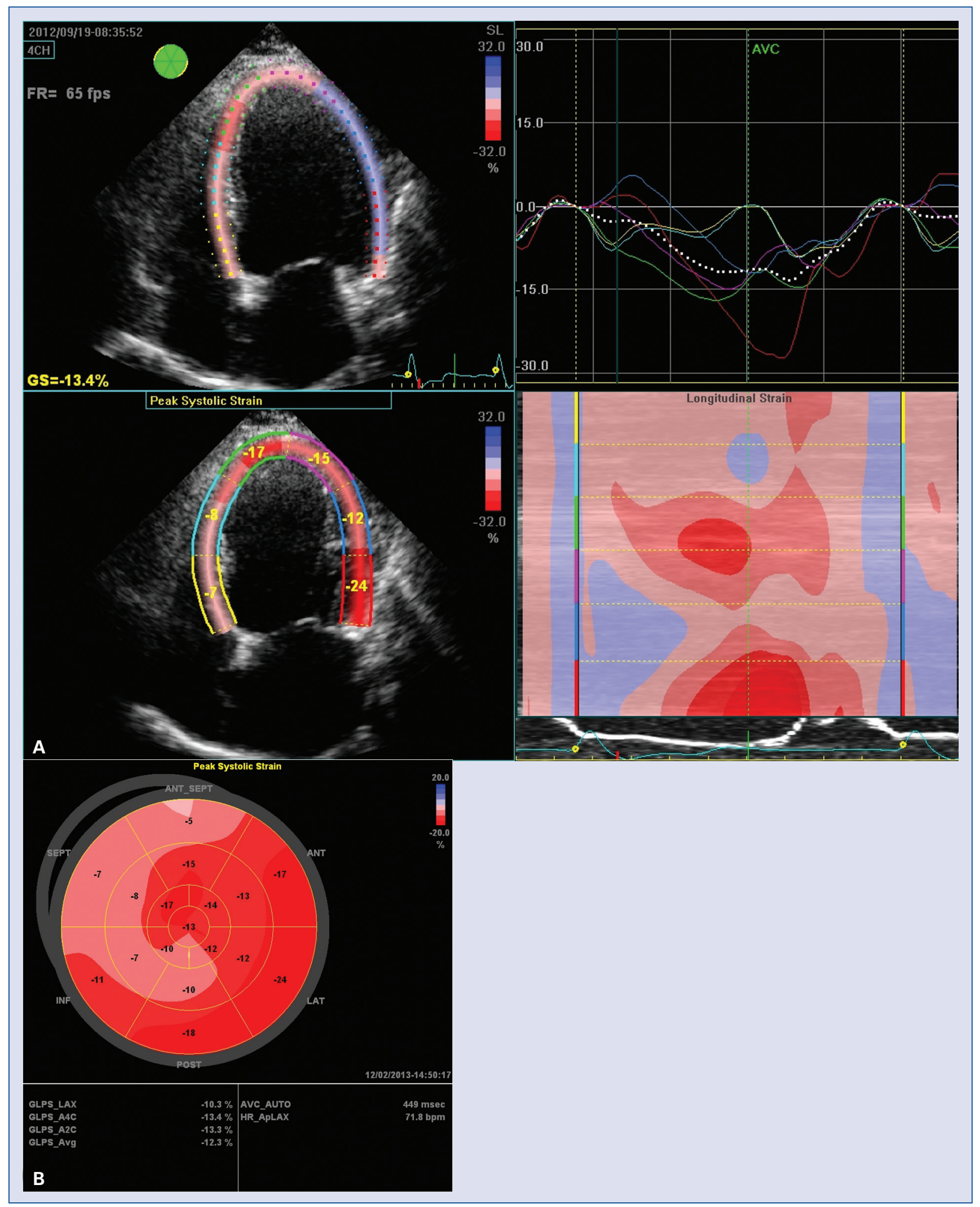

Figure 1. The endocardial border was traced manually in the end-diastolic frame. Graphical displays of deformation parameters for each segment were then generated automatically in apical 4 (A), 3 and 2 chamber views. Finally, left ventricular average global longitudinal strain for 18 segments (GLPS_Avg) was presented in bull's eye (B) scheme. 
performed to examine the relationship between hs-cTnT and other variables. Variables which showed significant correlation with hs-cTnT groups by univariate binary logistic regression analyses were tested with multiple regression analysis. Independent predictors of hs-cTnT above median were determined for both IHF and DCM groups. Reproducibility of echocardiographic parameters was assessed between measurements by coefficients of variation (standard deviation of differences between the repeated measurements divided by the mean value and expressed as percent). The intraobserver variability was calculated from 25 randomly selected subjects. Statistical analyses were performed with SPSS for Windows, version 20.0. P-values less than 0.05 were considered statistically significant.

\section{Results}

\section{Patient characteristics}

Clinical characteristics of patents are listed in Table 1 . The patient population consisted of $19 \mathrm{fe}-$ male, 41 male patients aged $56.3 \pm 13.9$ years. Thirty-three patients presented with IHF and 27 with DCM. Hs-cTnT was detectable ( $>0.003 \mathrm{ng} / \mathrm{mL}$ ) in all patients and $20(30 \%)$ patients had hs-cTnT concentrations of $>0.014 \mathrm{ng} / \mathrm{mL}$. Hs-cTnT concentrations were higher in males when log hs-cTnT values were compared $[-1.9 \pm 0.26$ for males $(\mathrm{n}=41)$ vs. $-2.2 \pm 0.24$ for females $(\mathrm{n}=19)$; $\mathrm{p}<0.001]$. Median values of hs-cTnT were $0.006 \mathrm{ng} / \mathrm{mL}$ for females and $0.011 \mathrm{ng} / \mathrm{mL}$ for males. Mean GFR was $88.8 \pm 29.1 \mathrm{~mL} / \mathrm{min}$ for the whole cohort, 11 (18\%) patients had GFR $<60 \mathrm{~mL} / \mathrm{min}$ and there was no significant difference between GFR values in patients with IHF and DCM. Hs-cTnT concentrations, EF, as well as aortic annulus diameters were higher in patients with IHF. However, LAVI was detected to be higher in patients with DCM. BNP values were not different statistically when compared for IHF and DCM (Table 2).

\section{Correlations of hs-cTnT with clinical variables and BNP}

When non-parametric correlations were analyzed hs-cTnT was found to be positively correlated with age, LV end-diastolic diameter (LVEDD), diameter of the aortic annulus, right ventricular and right atrial dimensions, E/Em lateral, BNP, and creatinine. It was also negatively correlated with Sm lateral and LV-GLS. However, there was no correlation between LWMI, GFR, LAVI and hs-cTnT (Table 3 ).
Table 1. Baseline characteristics of the study population.

\begin{tabular}{|c|c|}
\hline \multicolumn{2}{|l|}{ Clinical features } \\
\hline Age [years] & $56.3 \pm 14$ \\
\hline Sex (males) & $41(68 \%)$ \\
\hline Etiology (IHF) & $33(55 \%)$ \\
\hline Atrial fibrillation & $13(22 \%)$ \\
\hline Renal failure & $10(18 \%)$ \\
\hline Diabetes mellitus & $13(22 \%)$ \\
\hline Hypertension & $37(62 \%)$ \\
\hline Hyperlipidemia & $17(29 \%)$ \\
\hline Smoking & $13(22 \%)$ \\
\hline \multicolumn{2}{|l|}{ Pharmacologic treatment } \\
\hline Beta-blocker & $57(95 \%)$ \\
\hline ACEI/ARB & $49(82 \%)$ \\
\hline Furosemide & $39(65 \%)$ \\
\hline Spironolactone & $46(77 \%)$ \\
\hline Digoxin & $9(15 \%)$ \\
\hline Acetylsalicylic acid & $48(80 \%)$ \\
\hline OAD & $11(18 \%)$ \\
\hline Insulin & $4(7 \%)$ \\
\hline Statin & $15(25 \%)$ \\
\hline Nitrates & $8(13 \%)$ \\
\hline \multicolumn{2}{|l|}{ Echocardiographic parameters } \\
\hline \multicolumn{2}{|l|}{ Mitral regurgitation: } \\
\hline None & $4(6.7 \%)$ \\
\hline Mild & $34(56.7 \%)$ \\
\hline Moderate & $11(18.3 \%)$ \\
\hline Severe & $11(18.3 \%)$ \\
\hline LVEDD [cm] & $6.7 \pm 0.7$ \\
\hline LVESD [cm] & $5.5 \pm 0.7$ \\
\hline $\mathrm{EF}[\%]$ & $30.8 \pm 6.5$ \\
\hline GLS [\%] & $-7 \pm 2.9$ \\
\hline PAPs [mm Hg] & $39.2 \pm 11.5$ \\
\hline LAVI $\left[\mathrm{mL} / \mathrm{m}^{2}\right]$ & $42 \pm 17$ \\
\hline Aortic annulus [cm] & $3 \pm 0.3$ \\
\hline \multicolumn{2}{|l|}{ Laboratory findings } \\
\hline Blood urea nitrogen [mg/dL] & $39.2 \pm 14.7$ \\
\hline Potassium [mEq/L] & $4.6 \pm 0.4$ \\
\hline Sodium $[\mathrm{mEq} / \mathrm{L}]$ & $137 \pm 3.4$ \\
\hline $\mathrm{GFR}[\mathrm{ml} / \mathrm{min}]$ & $88.8 \pm 29.1$ \\
\hline Creatinine [mg/dL] & $1.03 \pm 0.3$ \\
\hline Haemoglobin $[\mathrm{g} / \mathrm{dL}]$ & $13.6 \pm 1.5$ \\
\hline BNP $[\mathrm{pg} / \mathrm{mLl}]$ & $215(17-1598)$ \\
\hline Hs-cTnT [ng/mL] & $0.011(0.003-0.051)$ \\
\hline
\end{tabular}


Table 2. Clinical and laboratory findings of patients with heart failure grouped according to etiology.

\begin{tabular}{|c|c|c|c|}
\hline Variable & Ischemic heart failure ( $n=33$ ) & Dilated cardiomyopathy $(n=27)$ & $\mathbf{P}$ \\
\hline \multicolumn{4}{|c|}{ Clinical and key laboratory findings } \\
\hline Age [years] & $59.0 \pm 9.5$ & $53.0 \pm 17.5$ & 0.093 \\
\hline Hs-cTnT [ng/mL] & $0.014(0.004-0.051)$ & $0.008(0.003-0.029)$ & $0.018 *$ \\
\hline BNP $[\mathrm{pg} / \mathrm{mL}]$ & 198 (19.4-1598) & $249.4(17.2-1540)$ & 0.345 \\
\hline Log hs-cTnT & $-1.9 \pm 0.3$ & $-2.1 \pm 0.3$ & $0.015^{*}$ \\
\hline Log BNP & $2.2 \pm 0.3$ & $2.3 \pm 0.4$ & 0.290 \\
\hline \multicolumn{4}{|c|}{ Echocardiographic parameters } \\
\hline GLS [\%] & $-6.8 \pm 3.0$ & $-7.2 \pm 3.0$ & 0.640 \\
\hline $\mathrm{EF}[\%]$ & $33 \pm 6$ & $29 \pm 7$ & $0.015^{*}$ \\
\hline LWMI $\left[\mathrm{g} / \mathrm{m}^{2}\right]$ & $180(39.7-312.5)$ & $166(99.6-310)$ & 0.694 \\
\hline LVEDD [cm] & $6.8 \pm 0.6$ & $6.6 \pm 0.7$ & 0.285 \\
\hline LVESD [cm] & $5.5 \pm 0.7$ & $5.6 \pm 0.7$ & 0.699 \\
\hline Aortic annulus [cm] & $3.0 \pm 0.3$ & $2.9 \pm 0.3$ & $0.017^{*}$ \\
\hline LAVI $\left[\mathrm{ml} / \mathrm{m}^{2}\right]$ & $38 \pm 15.2$ & $47 \pm 18.0$ & $0.039 *$ \\
\hline E mitral $[\mathrm{m} / \mathrm{s}]$ & $0.86 \pm 0.31$ & $0.92 \pm 0.31$ & 0.433 \\
\hline Em septum [m/s] & $0.053 \pm 0.016$ & $0.056 \pm 0.016$ & 0.388 \\
\hline Em lateral [m/s] & $0.071 \pm 0.025$ & $0.075 \pm 0.037$ & 0.656 \\
\hline E/Em septum & $16.7(6.7-43.3)$ & $14.8(10-47.5)$ & 0.882 \\
\hline E/Em lateral & $11(5.4-29.2)$ & $11.5(5-63.3)$ & 0.567 \\
\hline RVEDD [cm] & $3.4 \pm 0.5$ & $3.2 \pm 0.5$ & 0.218 \\
\hline $\mathrm{RA}[\mathrm{cm}]$ & $3.6 \pm 0.6$ & $3.4 \pm 0.5$ & 0.108 \\
\hline PAPs [mm Hg] & $38.9 \pm 13.3$ & $39.5 \pm 9.0$ & 0.858 \\
\hline TAPSE $[\mathrm{cm}]$ & $1.7 \pm 0.3$ & $1.7 \pm 0.5$ & 0.939 \\
\hline \multicolumn{4}{|c|}{ Other laboratory findings } \\
\hline GFR $[\mathrm{mL} / \mathrm{min}]$ & $90.0 \pm 30.1$ & $87.3 \pm 27.3$ & 0.727 \\
\hline Sodium [mEq/L] & $137 \pm 3.8$ & $137 \pm 3$ & 0.712 \\
\hline Potassium [mEq/L] & $4.6 \pm 0.5$ & $4.6 \pm 0.4$ & 0.613 \\
\hline BUN [mg/dL] & $41.8 \pm 13.9$ & $36.5 \pm 15.3$ & 0.161 \\
\hline Hemoglobin [g/dL] & $13.8 \pm 1.4$ & $13.5 \pm 1.7$ & 0.501 \\
\hline
\end{tabular}

Data are expressed as mean \pm SD or median (interquartile range); ${ }^{*} \mathrm{p}<0.05$; BNP — B-type natriuretic peptide; BUN - blood urea nitrogen; $\mathrm{E}$ mitral — peak early diastolic inflow velocity; EF — ejection fraction; Em — peak early diastolic tissue Doppler velocity; GFR - glomerular filtration rate; GLS - global longitudinal strain; Hs-cTnT — high sensitivity troponin T; LAVI — left atrial volume index; LVEDD - left ventricular end-diastolic diameter; LVESD - left ventricular end-systolic diameter; LWMI — left ventricular mass index; PAPs - systolic pulmonary artery pressure; RA — right atrium; RVEDD — right ventricular end diastolic diameter; TAPSE — tricuspid annular plane systolic excursion

\section{Predictors of hs-cTnT: DCM vs. IHF}

The patients were divided into two groups according to hs-cTnT median value; afterwards, univariate and multivariate logistic regression analyses were performed to estimate hs-cTnT above median.

In patients with IHF mitral $\mathrm{E}$ velocity, $\mathrm{E} / \mathrm{Em}$ lateral, Sm lateral and $\log B N P$ were univariate predictors of hs-cTnT above median. But only E/Em lateral was an independent predictor of hs-cTnT above median ( $\mathrm{p}=0.04$, HR: $1.2,95 \%$ CI $1-1.4)$. However in patient with DCM; LAVI and LV-GLS were included in multivariate model and LV-GLS was detected to be an independent predictor for
hs-cTnT above median ( $\mathrm{p}=0.03$, HR $0.7,95 \% \mathrm{CI}$ 0.4-1.0) (Table 4).

\section{Discussion}

In the present study we examined the clinical and echocardiographic predictors of hs-c $\operatorname{Tn} \mathrm{T}$ in patients with IHF vs. DCM. The novel finding of our study is that LV-GLS was an independent predictor of hs-cTnT in patients with DCM, but it was not even a univarite predictor in patients with IHF. Additionally, E/Em lateral was an independent predictor of hs-cTnT concentrations in patients with IHF. 
Table 3. Non-parametric correlations of hs-cTnT with other clinical variables.

\begin{tabular}{|c|c|c|}
\hline Variable & $\mathbf{r}$ & $\mathbf{p}$ \\
\hline Age & 0.28 & $0.033^{*}$ \\
\hline LVEDD & 0.26 & $0.044^{*}$ \\
\hline LVESD & 0.21 & 0.11 \\
\hline EF & -0.024 & 0.85 \\
\hline PW & 0.24 & 0.07 \\
\hline LA & 0.17 & 0.2 \\
\hline Aortic annulus & 0.37 & $0.004^{*}$ \\
\hline LAVI & -0.033 & 0.8 \\
\hline E mitral & 0.15 & 0.26 \\
\hline Em septal & -0.16 & 0.23 \\
\hline Em lateral & -0.16 & 0.21 \\
\hline E/Em septal & 0.16 & 0.21 \\
\hline E/Em lateral & 0.26 & $0.043^{*}$ \\
\hline Sm lateral & -0.41 & $0.001 *$ \\
\hline Sm septum & -0.1 & 0.47 \\
\hline RVEDD & 0.36 & $0.004^{*}$ \\
\hline RA & 0.26 & $0.043^{*}$ \\
\hline GLS & -0.32 & $0.013^{*}$ \\
\hline LWMI & 0.11 & 0.39 \\
\hline BNP & 0.28 & $0.029 *$ \\
\hline GFR & -0.15 & 0.25 \\
\hline Creatinine & 0.34 & $0.009 *$ \\
\hline Sodium & -0.13 & 0.34 \\
\hline Potassium & 0.17 & 0.18 \\
\hline Hemoglobin & -0.003 & 0.98 \\
\hline
\end{tabular}

${ }^{*} \mathrm{p}<0.05 ; \mathrm{BNP}$ - B-type natriuretic peptide; E mitral — peak early diastolic inflow velocity; EF — ejection fraction; Em - peak early diastolic tissue Doppler velocity; GFR - glomerular filtration rate; GLS - global longitudinal strain; Hs-cTnT — high sensitivity troponin T; LA - left atrium; LAVI - left atrial volume index; LVEDD - left ventricular end diastolic diameter; LVESD - left ventricular end-systolic diameter; LWMI - left ventricular mass index; PW posterior wall: RA — right atrium; RVEDD — right ventricular end-diastolic diameter; Sm - peak systolic tissue Doppler velocity
Several hypotheses, including subendocardial ischemia due to a mismatch between myocardial oxygen supply and demand, have been proposed as explanation for increased concentrations of cTnT in HF, but the mechanisms by which these features cause increase in troponin concentrations in the absence of an acute coronary syndrome remain uncertain. Higher troponin concentrations were observed in patients with HF of ischemic etiology, yet, consistent with our findings, elevated troponin concentrations were reported also in patients with non-ischemic HF $[9,10]$. An elevation in cardiac troponins can indicate the presence of myocyte injury or death. The pathophysiological factors that are thought to be responsible for ongoing myocyte injury or cell death include excessive adrenergic stimulation through renin-angiotensin-aldosterone or endothelin signalling pathways, abnormalities in calcium handling, inflammatory cytokines, nitric oxide, and oxidative or mechanical stress [15].

However, the elevations of cTnT were detectable only in a small fraction of patients with stable chronic HF because previous studies used conventional troponin $\mathrm{T}$ assays for the lower detection limit of $0.01 \mathrm{ng} / \mathrm{mL}$. Recently, the highly sensitive assay for cTnT has been developed and made it possible to measure concentrations about 10 -fold lower than the lower detection limit of the previous conventional assay [12]. Thanks to the highly sensitive assay, circulating cTnT has been detected in a large population of patients with HF, and it provided prognostic information about previously undetectable concentrations regardless of their etiology $[2,16]$. The prognostic significance of hs-cTnT assay was reported a continuous variable

Table 4. Univariate and multivariate relations for prediction of hs-cTnT concentrations.

\begin{tabular}{|c|c|c|c|c|c|c|}
\hline & \multicolumn{3}{|c|}{ Univariate } & \multicolumn{3}{|c|}{ Multivariate } \\
\hline & HR & $95 \% \mathrm{Cl}$ & $\mathbf{P}$ & HR & $95 \% \mathrm{Cl}$ & $\mathbf{P}$ \\
\hline \multicolumn{7}{|c|}{ Ischemic heart failure } \\
\hline E mitral & 14 & $1-194$ & 0.043 & NS & & \\
\hline E/Em septal & 1.1 & $1-1.2$ & 0.061 & NS & & \\
\hline E/Em lateral & 1.2 & $1-1.4$ & 0.040 & 1.2 & $1-1.4$ & 0.04 \\
\hline Sm lateral & 1.5 & $1-1.9$ & 0.040 & NS & & \\
\hline Log BNP & 8 & $1-62.8$ & 0.049 & NS & & \\
\hline \multicolumn{7}{|c|}{ Non-ischemic cardiomyopathy } \\
\hline Male sex & 0.25 & $0.05-1.2$ & 0.088 & NS & & \\
\hline LAVI & 0.9 & $0.9-1$ & 0.045 & NS & & \\
\hline Sm lateral & 0.95 & $0.9-1$ & 0.068 & NS & & \\
\hline GLS & 0.7 & $0.4-1$ & 0.030 & 0.7 & $0.4-1$ & 0.03 \\
\hline
\end{tabular}

BNP - B-type natriuretic peptide; $\mathrm{Cl}$ — confidence interval; E mitral — peak early diastolic inflow velocity; Em — peak early diastolic tissue Doppler velocity; GLS — global longitudinal strain; HR — hazard ratio; Hs-cTnT — high sensitivity troponin T; LAVI — left atrial volume index; NS - not significant; Sm - peak systolic tissue Doppler velocity 
in patients with chronic HF, in contrast to the conventional cTnT assay, often described as a positive or negative categorical variable [2]. Furthermore, Miller et al. [17, 18] reported that a decrease in cTnT concentrations to normal values was associated with a decrease in risks of death or cardiac transplantation, whereas a normalization of BNP concentrations did not change these risks. Even small elevations of cTnT concentrations might be an indication for more aggressive treatment.

Previously, hs-cTnT $>0.003 \mathrm{ng} / \mathrm{mL}$ was associated with male sex, older age, history of HF, lower renal function, and a greater LVM [19]. Male patients in our study population also had higher hs-cTnT concentrations than females and their age was correlated with hs-cTnT concentrations consistent with these findings, but GFR and LWMI were not related to hs-cTnT concentrations. The reason for this may be the exclusion of patients with creatinine concentrations of $>2 \mathrm{mg} / \mathrm{dL}$ and relatively preserved renal function of our study population. In the mentioned study electrocardiographic LV hypertrophy was an independent predictor of hs-cTnT, although we evaluated echocardiographic LV hypertrophy.

$\mathrm{E} / \mathrm{Em}$ lateral was an independent predictor of higher hs-cTnT concentrations in patients with IHF. Since E/Em lateral predicts pulmonary capillary wedge pressure and is less affected from coronary artery disease than E/Em septal, worsening subendocardial demand-supply relationship as a result of diastolic dysfunction may be the dominating pathophysiologic mechanism responsible for cTnT release. Recently, Eggers et al. [20] examined 103 stable patients with LV dysfunction and found that patients with higher hs-cTnT (> $13.5 \mathrm{ng} / \mathrm{L}$ ) had higher pulmonary capillary wedge pressure, mean right atrial pressure, mean pulmonary artery pressure, and pulmonary vascular resistance, but lower cardiac index than patients with lower hs-cTnT ( $\leq 13.5 \mathrm{ng} / \mathrm{L})$.

Our study showed that in patients with DCM LV-GLS was an independent predictor of hs-cTnT concentrations. LV mechanics is a complex, coordinated action involving longitudinal contraction, circumferential shortening, and radial thickening. Additionally, many studies have shown that measurement of LV longitudinal motion by the mitral annular excursion or velocities has been a good index of $\mathrm{LV}$ function and also a predictor of prognosis in HF [21-25]. Recently decreased longitudinal mechanics (LV-GLS) was associated with increased cardiac events of both ischemic and non-ischemic HF during 5 years of follow-up. Moreover, LV-GLS was reported to have significant incremental value over conventional clinical and echocardiographic parameters, including longitudinal velocity [7]. We speculate that LV-GLS may be a more valuable prognostic marker in DCM rather than IHF.

\section{Limitations of the study}

The major limitation of the present study is the relatively low number of patients included. Therefore, our results are only hypothesis generating and require confirmation in larger studies. The hs-cTnT concentrations were measured only once at presentation to outpatient clinic, so acute coronary syndrome as a cause of hs-c TnT release might be overlooked. Besides, we did not perform invasive testing or long term rhythm Holter follow-up. It is therefore convincible that to certain extent clinically undetected variation of phenotype such as intermittent change in rhythm or change of loading conditions might confound the findings.

\section{Conclusions}

In contrary to acute coronary syndromes, an objective risk-stratification process for the evaluation of patients with $\mathrm{HF}$ is lacking. Early risk stratification may help identify patients who are likely to receive the greatest benefit from early intensive therapy. Since DCM and IHF are distinct clinical entities both presenting with HF, it may be reasonable to use different risk stratification measures.

\section{Conflict of interest: none declared}

\section{References}

1. Thygesen K, Alpert JS, Jaffe AS, Simoons ML, Chaitman BR, White HD; Joint ESC/ACCF/AHA/WHF Task Force for the Universal Definition of Myocardial Infarction. Third universal definition of myocardial infarction. Circulation, 2012; 126: 2020-2035.

2. Latini R, Masson S, Anand IS et al; Val-HeFT Investigators. Prognostic value of very low plasma concentrations of troponin $\mathrm{T}$ in patients with stable chronic heart failure. Circulation, 2007; 116: $1242-129$.

3. Omland T, de Lemos JA, Sabatine MS et al; Prevention of Events with Angiotensin Converting Enzyme Inhibition (PEACE) Trial Investigators. A sensitive cardiac troponin $\mathrm{T}$ assay in stable coronary artery disease. N Engl J Med, 2009; 361: 2538-2547.

4. Masson S, Anand I, Favero C et al; Valsartan Heart Failure Trial (Val-HeFT) and Gruppo Italiano per lo Studio della Sopravvivenza nell'Insufficienza Cardiaca-Heart Failure (GISSI-HF) Investigators. Serial measurement of cardiac troponin T using a highly sensitive assay in patients with chronic heart failure: Data from 2 large randomized clinical trials. Circulation, 2012; 125: 280-288.

5. Yamashita T, Seino Y, Ogawa A et al. N-terminal pro-BNP is a novel biomarker for integrated cardio-renal burden and early 
risk stratification in patients admitted for cardiac emergency. J Cardiol, 2010; 55: 377-383.

6. Sato Y, Kita T, Takatsu Y, Kimura T. Heart. Biochemical markers of myocyte injury in heart failure. Heart, 2004; 90: 1110-1113.

7. Motoki H, Borowski AG, Shrestha K et al. Incremental prognostic value of assessing left ventricular myocardial mechanics in patients with chronic systolic heart failure. J Am Coll Cardiol, 2012; 60: 2074-2081.

8. Mignot A, Donal E, Zaroui A, et al. Global longitudinal strain as a major predictor of cardiac events in patients with depressed left ventricular function: A multicenter study. J Am Soc Echocardiogr, 2010; 23: 1019-1024.

9. Bart BA, Shaw LK, McCants CB Jr, et al. Clinical determinants of mortality in patients with angiographically diagnosed ischemic or nonischemic cardiomyopathy. J Am Coll Cardiol, 1997; 30: 1002-1008.

10. Likoff MJ, Chandler SL, Kay HR. Clinical determinants of mortality in chronic congestive heart failure secondary to idiopathic dilated or to ischemic cardiomyopathy. Am J Cardiol, 1987; 59: 634-638.

11. McMurray JJ, Adamopoulos S, Anker SD et al. ESC Committee for Practice Guidelines. ESC guidelines for the diagnosis and treatment of acute and chronic heart failure 2012: The Task Force for the Diagnosis and Treatment of Acute and Chronic Heart Failure 2012 of the European Society of Cardiology. Developed in collaboration with the Heart Failure Association (HFA) of the ESC. Eur J Heart Fail, 2012; 14: 803-869.

12. Apple FS, Collinson PO. Analytical characteristics of high-sensitivity cardiac troponin assays. Clin Chem, 2012; 58: 54-61.

13. Lang RM, Bierig M, Devereux RB et al. Recommendations for chamber quantification: a report from the American Society of Echocardiography's Guidelines and Standards Committee and the Chamber Quantification Writing Group. J Am Soc Echocardiogr, 2005; 18: 1440-1463.

14. Sato $\mathrm{H}$, Watanabe $\mathrm{N}$, Adachi $\mathrm{T}$ et al. Factors influencing left atrial volume in treated hypertension. J Cardiol. 2012; 60: 133-138.

15. Kociol RD, Pang PS, Gheorghiade M, Fonarow GC, O'Connor CM, Felker GM. Troponin elevation in heart failure prevalence, me- chanisms, and clinical implications. J Am Coll Cardiol, 2010; 56: 1071-1078.

16. Kawahara C, Tsutamoto T, Nishiyama K et al. Prognostic role of high-sensitivity cardiac troponin $\mathrm{T}$ in patients with nonischemic dilated cardiomyopathy. Circ J, 2011; 75: 656-661.

17. Miller WL, Hartman KA, Burritt MF et al. Serial biomarker measurements in ambulatory patients with chronic heart failure: The importance of change over time. Circulation, 2007; 116: 249-257.

18. Miller WL, Hartman KA, Burritt MF, Grill DE, Jaffe AS. Profiles of serial changes in cardiac troponin $\mathrm{T}$ concentrations and outcome in ambulatory patients with chronic heart failure. J Am Coll Cardiol, 2009; 54: 1715-1721.

19. Sato Y, Yamamoto E, Sawa T et al. High-sensitivity cardiac tropo$\operatorname{nin} \mathrm{T}$ in essential hypertension. J Cardiol, 2011; 58: 226-231.

20. Eggers KM, Nygren M, Venge P, Jernberg T, Wikström BG. High sensitive troponin $\mathrm{T}$ and $\mathrm{I}$ are related to invasive hemodynamic data and mortality in patients with left-ventricular dysfunction and precapillary pulmonary hypertension. Clin Chim Acta, 2011; 412: $1582-1588$.

21. Sveälv BG, Olofsson EL, Andersson B. Ventricular long-axis function is of major importance for long-term survival in patients with heart failure. Heart, 2008; 94: 284-289.

22. Simonson JS, Schiller NB. Descent of the base of the left ventricle: An echocardiographic index of left ventricular function. J Am Soc Echocardiogr, 1989; 2: 25-35.

23. Willenheimer R, Cline C, Erhardt L, Israelsson B. Left ventricular atrioventricular plane displacement: An echocardiographic technique for rapid assessment of prognosis in heart failure. Heart, 1997; 78: 230-236.

24. Nikitin NP, Loh PH, Silva RD et al. Prognostic value of systolic mitral annular velocity measured with Doppler tissue imaging in patients with chronic heart failure caused by left ventricular systolic dysfunction. Heart, 2006; 92: 775-779.

25. Wang M, Yip G, Yu CM et al. Independent and incremental prognostic value of early mitral annulus velocity in patients with impaired left ventricular systolic function. J Am Coll Cardiol, 2005; 45: 272-277. 